\title{
LARGE CONTRACTIBLE SUBGRAPHS OF A 3-CONNECTED GRAPH
}

\author{
DMITRI V. KARPOV ${ }^{1}$ \\ St. Petersburg Department of V.A. Steklov Institute of Mathematics \\ of the Russian Academy of Sciences and St. Petersburg University \\ St. Petersburg, Russia \\ e-mail: dvk0@yandex.ru
}

\begin{abstract}
Let $m \geq 5$ be a positive integer and let $G$ be a 3 -connected graph on at least $2 m+1$ vertices. We prove that $G$ has a contractible set $W$ such that $m \leq|W| \leq 2 m-4$. (Recall that a set $W \subset V(G)$ of a 3-connected graph $G$ is contractible if the graph $G(W)$ is connected and the graph $G-W$ is 2-connected.) A particular case for $m=4$ is that any 3-connected graph on at least 11 vertices has a contractible set of 5 or 6 vertices.
\end{abstract}

Keywords: connectivity, 3-connected graph, contractible subgraph.

2010 Mathematics Subject Classification: 05C40.

\section{REFERENCES}

[1] W.T. Tutte, Connectivity in Graphs (Univ. Toronto Press, 1966).

[2] W. Hohberg, The decomposition of graphs into $k$-connected components, Discrete Math. 109 (1992) 133-145.

doi:10.1016/0012-365X(92)90284-M

[3] W. Mader, On vertices of degree $n$ in minimally $n$-connected graphs and digraphs, Combinatorics, Paul Erdős is Eighty 2 (1996) 423-449.

[4] W. McCuaig and K. Ota, Contractible triples in 3-connected graphs, J. Comb. Theory Ser. B 60 (1994) 308-314. doi:10.1006/jctb.1994.1022

[5] M. Kriesell, Contractible subgraphs in 3-connected graphs, J. Comb. Theory Ser. B 80 (2000) $32-48$. doi: $10.1006 /$ jctb. 2000.1960

\footnotetext{
${ }^{1}$ This research is supported by the Government of the Russian Federation (Grant No. 14.Z50.31.0030).
} 
[6] M. Kriesell, On small contractible subgraphs in 3-connected graphs of small average degree, Graphs Combin. 23 (2007) 545-557.

doi:10.1007/s00373-007-0749-5

[7] F. Harary, Graph Theory (Addison-Wesley, 1969). doi:10.21236/AD0705364

[8] D.V. Karpov and A.V. Pastor, On the structure of a $k$-connected graph, J. Math. Sci. 113 (2003) 584-597. doi:10.1023/A:1021146226285

[9] D.V. Karpov and A.V. Pastor, The structure of a decomposition of a triconnected graph, J. Math. Sci. 184 (2012) 601-628. doi:10.1007/s10958-012-0885-1

[10] D.V. Karpov, Blocks in k-connected graphs, J. Math. Sci. 126 (2005) 1167-1181. doi:10.1007/s10958-005-0084-4

[11] D.V. Karpov, Cutsets in a k-connected graph, J. Math. Sci. 145 (2007) 4953-4966. doi:10.1007/s10958-007-0330-z

[12] D.V. Karpov, The decomposition tree of a biconnected graph, J. Math. Sci. 204 (2015) 232-243. doi:10.1007/s10958-014-2198-z

[13] D.V. Karpov, Minimal biconnected graphs, J. Math. Sci. 204 (2015) 244-257. doi:10.1007/s10958-014-2199-y

Received 29 May 2017

Revised 18 August 2018

Accepted 20 August 2018 